\title{
Do you remember your sad face? The roles of negative cognitive style and sad mood
}

\author{
Corrado Caudek $^{1,2}$ and Alessandra Monni ${ }^{1}$ \\ ${ }^{1}$ Dipartimento di Psicologia, Università degli Studi di Firenze, Firenze, Italy \\ ${ }^{2}$ Center for Neuroscience and Cognitive Systems@UniTn, Istituto Italiano di Tecnologia, \\ Rovereto, Italy
}

\begin{abstract}
We studied the effects of negative cognitive style, sad mood, and facial affect on the self-face advantage in a sample of 66 healthy individuals (mean age 26.5 years, range $19-47$ years). The sample was subdivided into four groups according to inferential style and responsivity to sad mood induction. Following a sad mood induction, we examined the effect on working memory of an incidental association between facial affect, facial identity, and head-pose orientation. Overall, head-pose recognition was more accurate for the self-face than for nonself face (self-face advantage, SFA). However, participants high in negative cognitive style who experienced higher levels of sadness displayed a stronger SFA for sad expressions than happy expressions. The remaining participants displayed an opposite bias (a stronger SFA for happy expressions than sad expressions), or no bias. These findings highlight the importance of trait-vulnerability status in the working memory biases related to emotional facial expressions.
\end{abstract}

Keywords: Self-face advantage; Working memory; Cognitive bias; Negative cognitive style; Emotion; Sad mood induction.

Many studies have found that a negative cognitive style, defined as the tendency to attribute negative life events to stable, global, and internal causes, in interaction with stressful life events, represents a robust risk factor for depression (Alloy et al., 2000; Alloy, Abramson, Keyser, Gerstein, \& Sylvia, 2008). Depressed individuals show a memory bias towards negative information and/or away from positive information (Matt, Vazquez, \& Campbell, 1992). But are healthy individuals with a negative cognitive style not only more likely to become depressed, but also more likely to experience a negative memory bias than individuals with more positive cognitive style?

A relation between negative cognitive style and maladaptive information processing in healthy individuals has been reported, for example, by Romens, MacCoon, Ambramson, and Pollak (2011). By using an attentional-blink (AB) technique, ${ }^{1}$ they showed that a negative cognitive style can alter the automatic allocation of attention to emotional information. Romens et al. found that, under conditions of limited attentional resources, healthy participants high in negative cognitive style in whom a negative mood was induced were more likely than participants with low or moderate negative cognitive style to detect negative attribution words (e.g., "failure"), compared to nonself-relevant negative

\footnotetext{
${ }^{1}$ The $\mathrm{AB}$ refers to the phenomenon in which participants show a reduced ability to report the second of two masked targets when it appears within $200-500 \mathrm{~ms}$ of the first.
}

Address correspondence to: Corrado Caudek, Dipartimento di Psicologia, Università degli Studi di Firenze, Via di San Salvi 12, Complesso di S. Salvi-Padiglione 26, 50135 Firenze, Italy. E-mail: corrado.caudek@unifi.it

We thank Claudio Sica for helpful discussions, and Laura Balzano, Carlotta Damiani, and Sibilla Vanni for their help in developing the stimuli and collecting data. 
(e.g., "danger") or neutral (e.g., "fabric") words. These data have been interpreted as indicating that a negative cognitive style can modulate the saliency of stimuli that are relevant to negative self-attributions.

Drawing on cognitive diathesis-stress theories (Disner, Beevers, Haigh, \& Beck, 2011; Ingram, Atchley, \& Segal, 2011), in the present study we used a negative mood-induction (MI) procedure to prime maladaptive information processing prior to the assessment of Working Memory (WM) performance for emotional faces (the selfface and a stranger's face) in nondepressed individuals. According to diathesis-stress models of depression, vulnerable individuals possess maladaptive cognitive schemas that distinguish them from nonvulnerable individuals. When activated by an external or internal stressor, these latent negative self-schemas generate maladaptive cognitive processes, such as negative interpretative, attentional, or memory biases (Abramson et al., 2002; Scher, Ingram, \& Segal, 2005). WM biases related to emotional processing are particularly important because they have been linked to the development and perpetuation of mood disorders (Gotlib \& Joormann, 2010).

Previous research indicates that, when self cues are present (such as a picture of the participant's own face), enhanced processing in the form of a memory advantage occurs, even if explicit instructions related to the self are not provided (e.g., Kesebir \& Oishi, 2010). Therefore, we expected that participants would demonstrate better WM recognition for the self-face than for stranger's faces (Ma \& Han, 2009, 2010). Importantly, the stimulus set of the present experiment included the sad self-face, because negative information related to the self, as opposed to negative information in general, provides the most sensitive test of biased memory in vulnerable (and depressed) individuals (Ramel et al., 2007; Werner-Seidler \& Moulds, 2012; Wisco, 2009). The purpose of our study was to relate the selfface advantage to facial affect, negative cognitive style, and sad mood. To our knowledge, no data have been published on abnormal memory for emotional faces when participants are asked to memorise images of their own face.

We considered the healthy individuals of our sample to be at "low or high cognitive risk" depending on their scores on the Cognitive Style Questionnaire (CSQ; Alloy et al., 2000; Haeffel et al., 2008). We also assumed that mood induction would precipitate maladaptive information processing only if participants had experienced a strong mood shift. By crossing "cognitive risk" (low, high) with individual responsivity to mood induction (weak, strong), we divided the participants into four groups (see Table 1).

In the encoding display, four photographs were shown. Two pictures depicted the participant's own face and two pictures portrayed a stranger's face, with happy and sad expressions. Pictures of the self-face were used in the attempt to heighten self-focus as an activating agent for latent negative self-schemas (Hedlund \& Rude, 1995). Participants provided old/new judgements about whether a successively presented probe face had the same head-pose orientation as in the encoding display (Figure 1). This procedure allowed us to measure the impact of self-referencing on unintentional encoding of affective facial expressions. Incidental encoding is less susceptible to conscious explicit processing, whereby individuals can alter their more automatic, schema-driven responses (Haeffel et al., 2007). The following three hypotheses were formulated.

Hypothesis 1: Individuals at high cognitive risk with a strong response to sad mood induction will display a stronger self-face advantage for sad expressions than happy expressions. In fact,

TABLE 1

Participant characteristics

\begin{tabular}{|c|c|c|c|c|}
\hline & $\begin{array}{c}h C R s R \\
(n=16)\end{array}$ & $\begin{array}{l}h C R w R \\
(n=17)\end{array}$ & $\begin{array}{c}l C R s R \\
(n=19)\end{array}$ & $\begin{array}{l}l C R w R \\
(n=14)\end{array}$ \\
\hline Age & $26.8(1.6)$ & $27.1(1.3)$ & $25.7(0.3)$ & $26.7(1.1)$ \\
\hline $\begin{array}{l}\text { Gender ratio } \\
(\mathrm{F} / \mathrm{M})\end{array}$ & $14 / 2$ & $10 / 7$ & $16 / 3$ & $10 / 4$ \\
\hline BDI-II & $\begin{array}{c}9.8(2.2) \\
{[3.0,35.0]}\end{array}$ & $\begin{array}{l}13.9(1.7) \\
{[4.0,31.0]}\end{array}$ & $\begin{array}{c}5.2(1.2) \\
{[0.0,17.0]}\end{array}$ & $\begin{array}{c}9.1(2.0) \\
{[0.0,25.0]}\end{array}$ \\
\hline CSQ & $\begin{array}{c}4.2(0.1) \\
{[3.7,5.4]}\end{array}$ & $\begin{array}{c}4.1(0.1) \\
{[3.7,5.1]}\end{array}$ & $\begin{array}{c}3.1(0.1) \\
{[2.1,3.6]}\end{array}$ & $\begin{array}{c}3.2(0.1) \\
{[2.0,3.6]}\end{array}$ \\
\hline RRS & $\begin{array}{c}36.7(2.9) \\
{[22.0,68.0]}\end{array}$ & $\begin{array}{r}43.4(2.9) \\
{[28.0,74.0]}\end{array}$ & $\begin{array}{r}30.2(1.4) \\
{[22.0,42.0]}\end{array}$ & $\begin{array}{r}38.6(2.5) \\
{[25.0,57.0]}\end{array}$ \\
\hline VAS-dif & $7.1(0.3)$ & $2.5(0.3)$ & $6.8(0.3)$ & $3.0(0.4)$ \\
\hline
\end{tabular}

BDI-II is Beck Depression Inventory Second Edition. CSQ is Cognitive Style Questionnaire. RRS is Ruminative Response Scale. VAS-dif is the difference between the postinduction and the preinduction mood checks measured with the Visual Analogue Scale. hCRsR: participants at high cognitive risk with a strong response to sad mood induction; hCRwR: participants at high cognitive risk with a weak response to sad mood induction; lCRsR: participants at low cognitive risk with a strong response to sad mood induction; lCRwR: participants at low cognitive risk with a weak response to sad mood induction. $S D$ is shown in parentheses. The range of values is indicated in square brackets. 


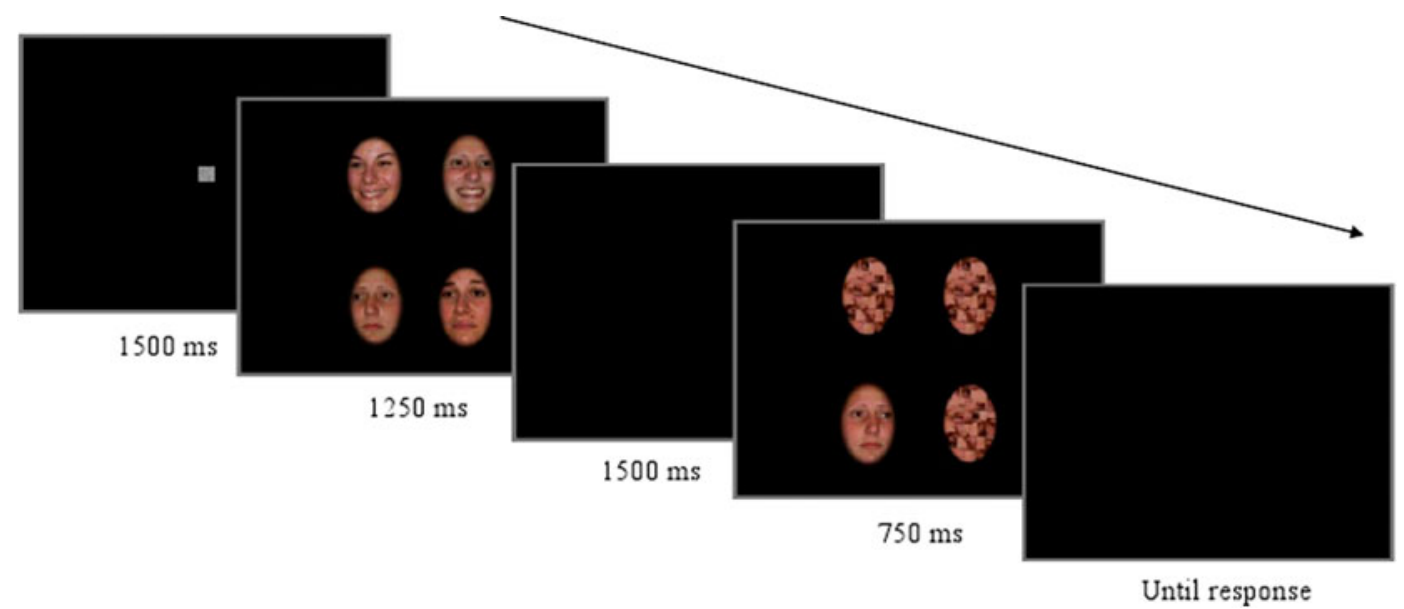

Figure 1. An example trial. After a fixation mark, an encoding display containing four faces, a blank screen (ISI =1500 ms), a memory probe, and another blank screen were presented sequentially. The participants reported whether the probe face had the same head-pose orientation as the spatially corresponding face in the encoding display. "New" faces differed from "old" faces in terms of head orientation $\left(30^{\circ}\right.$ right or left), whereas face identity (self-face or stranger's face) and facial expression (happy or sad) remained constant.

cognitive diathesis-stress models predict that, when activated, latent negative schemas generate a mood-congruent memory bias that causes negative information to become more salient and accessible, especially when it refers to the self. ${ }^{2}$

Hypothesis 2: Individuals at high cognitive risk with a weak response to sad mood induction will display a stronger self-face advantage for happy expressions than sad expressions. If negative self-schemas are dormant (not activated by a sufficiently strong emotional challenge), dysfunctional processing will not occur (Bistricky, Ingram, \& Atchley, 2011; Scher et al., 2005). In these conditions, we expect to find the same selfpositivity bias typically exhibited by nonvulnerable individuals (Watson, Dritschel, Obonsawin, \& Jentzsch, 2007).

Hypothesis 3: Individuals at low cognitive risk will display a stronger self-face advantage for happy expressions than sad expressions. If latent negative self-schemas are absent, we expect to find a self-positivity bias because resilient individuals tend to show an overoptimistic cognitive bias which provides motivational and moodbuffering benefits (Forgas \& East, 2008). We have no predictions concerning the relation between responsivity to sad MI and strength of the selfface advantage in individuals at low cognitive risk.

\footnotetext{
${ }^{2}$ When using lists of affective words, evidence of a moodcongruent memory bias in depressed and dysphoric individuals is especially evident for words considered in relation to the self (e.g., Hertel, 2004).
}

A memory bias towards the happy self-face is adaptive, because it may protect against negative mood and loss of self-esteem. Conversely, a memory bias towards the sad self-face is maladaptive, because it may result in a distorted mental representation of the social environment and it may contribute to negative self-appraisal and low self-concept.

\section{METHOD}

\section{Participants}

Sixty-six Caucasian undergraduate students (16 males, 50 females; mean age: 26.5 years; $S D: 4.5$; range: $19-47$ years) of the University of Firenze (Italy), with no history of neurological or psychiatric disorders, volunteered to take part in the current study. Participants were divided by median split into four groups based on their scores on the Cognitive Style Questionnaire (CSQ; Alloy et al., 2000) and on their response to sad MI: (1) high Cognitive Risk strong Response to sad MI ( $h C R s R, n=16)$, (2) high Cognitive Risk weak Response to sad MI ( $h C R w R, n=17)$, (3) low Cognitive Risk strong Response to sad MI (lCRsR, $n=19)$, (4) low Cognitive Risk weak Response to sad MI $(l C R w R, n=14)$. All participants gave informed written consent prior to taking part in the study, but were not informed of the true purpose of the study until the debriefing session. The study was approved by the Departmental Ethics Committee, Dipartimento di Psicologia, Università degli Studi di Firenze, Italy. 


\section{Measures}

The Beck Depression Inventory-II (BDI-II; Beck, Steer, \& Brown, 1996) is a well-validated 21-item self-report inventory of cognitive, affective, and neurovegetative depressive symptoms experienced in the past 2 weeks. We used the Italian version of the BDI-II (Ghisi, Flebus, Montano, Sanavio, \& Sica, 2006; Sica \& Ghisi, 2007). In the present study, the $\alpha$ coefficient (Cronbach's alpha) was .93, confirming internal consistency reliability.

The Cognitive Style Questionnaire (CSQ; Alloy et al., 2000) is a 48-item self-report measure of cognitive and behavioural coping strategies. Participants rate the probable consequences of 12 negative events (six interpersonal and six achievement events) and the implications of each event for the self-concept. The participants' inferences regarding the cause, consequence, and self-worth implications of each hypothetical negative event are assessed to measure the three components featured in the hopelessness theory of depression (Abramson, Metalsky, \& Alloy, 1989). The CSQ was translated into Italian as a part of a more comprehensive study on the role of cognitive variables in psychological disorders, and in particular in depression (Sica, Caudek, Chiri, Ghisi, \& Marchetti, 2012). In the present study, the internal consistency $(\alpha)$ coefficients were .81, .93, and .94 for the "Cause", "Consequence", and "Selfworth implications" subscales, respectively.

The Visual Analogue Scale (VAS; McCormack, Horne, \& Sheather, 1988) is a self-report device used to measure respondents' perceptions of their own mood (e.g., Scherrer \& Dobson, 2009). Participants rate their mood by placing a vertical mark on a $100 \mathrm{~mm}$ line, marked with "Not at all sad" on the left end and "Extremely sad" on the right. A score from 0 to 100 is derived by measuring the distance of the mark from the left pole. Participants completed the VAS before and then after the mood induction. VAS-dif was computed by subtracting the postinduction mood check from its baseline (e.g., Watkins, Teasdale, \& Williams, 2003).

The Ruminative Response Scale (RRS; NolenHoeksema \& Morrow, 1993) of the Response Styles Questionnaire includes 22 items describing the tendency to ruminate in response to depressed mood. The RRS assesses responses to dysphoric mood that are focused on the self, on symptoms, or on possible consequences and causes of moods.
We used the Italian version of the RRS (Palmieri, Gasparre, \& Lanciano, 2007). In the present study, the internal consistency $(\alpha)$ coefficients were .84 , .91 , and .88 for the "Brooding", "Reflection", and "Depression" subscales, respectively.

\section{Sad mood induction}

Participants were instructed to listen to Prokofiev's Alexander Nevsky: Russia under the Mongolian Yoke played at half speed (Rowe, Hirsh, \& Anderson, 2007) and to recall three experiences that made them feel lonely, rejected, defeated, or hurt (Singer \& Dobson, 2007). Immediately after they had finished listening to the music, participants were instructed to watch a video clip in order to increase their emotional engagement and to maintain their sad mood (Jallais \& Gilet, 2010). The video was a 3-minute scene of a boy crying over his father's death from the movie The Champ (Lovell \& Zeffirelli, 1979). Previous studies have tested the reliability of this clip to elicit sadness (Hewig et al., 2005). Each participant was alone while undergoing the mood induction.

\section{Stimuli}

In a pretest session, a total of 16 pictures was taken for each participant's positive and negative facial expressions, with a $30^{\circ}$ left or a $30^{\circ}$ right head orientation. Happy and sad expressions resulted from instructions to the participants to display prototypical, strong expressions of these emotions. To select the images that best represented each of the two facial expressions, the two authors and three undergraduate students rated the intensity of the facial expression of each picture on a Likert scale ranging from -7 (sad) to 0 (neutral) to +7 (happy) (Jermann, Van der Linden, \& D'Argembeau, 2008). A final set of eight happy and eight sad pictures was selected for each participant by choosing the pictures that obtained the highest positive and negative scores on the intensity scale. The pictures used in each trial of the experiment were randomly selected from these two final sets.

The encoding display consisted of four faces: two pictures of the participant and two picture of a stranger of the same sex, race, and approximately the same age. To control for low-level differences in visual salience, each participant was randomly coupled with another participant. 
Within each pair, the self images of one participant were used as the nonself images of the other. Each of the two facial identities displayed a happy and a sad facial expression. All faces were cropped to remove external features by the application of the same oval mask and were presented on a black background.

Face images were shown with a $30^{\circ}$ left or a $30^{\circ}$ right head orientation. Each of the 16 possible combinations of head-pose orientation in the encoding display was presented with the same probability in the course of the experiment. The relative spatial positions (top left, top right, bottom left, bottom right) of the four faces (happy selfface, happy nonself face, sad self-face, sad nonself face) were determined randomly on each trial.

"New" probe faces were generated by reflection about a vertical axis. Therefore, a "new" probe face was identical to the spatially corresponding face in the encoding display, except for the head-pose orientation $\left(30^{\circ}\right.$ left rather than $30^{\circ}$ right, or vice versa). The probe display consisted of four items: the probe face and three scrambled face photographs (see Figure 1).

\section{Procedure}

During an initial session that took place not more than 2 days before the experiment, participants completed a battery of three self-report scales: BDI-II, CSQ, and RRS. In the experimental sessions, prior to the mood inductions, participants rated their baseline mood with the VAS, for which they were instructed to focus on how they were feeling at that moment. They were then asked to get into a negative mood state using the music, the imagery suggested by the mood induction procedure and the movie, and to stay in that state until the whole session was completed. Following the induction, the mood check was administered again. Then the participants completed the experiment.

The trial sequence is illustrated in Figure 1. The experiment comprised 512 trials (256 "new" and 256 "old") randomly mixed up across participants. Participants were instructed to respond "same" with a keypress if the probe matched the spatially corresponding face in the encoding display in terms of head-pose orientation, and "different" otherwise. No feedback was provided for correct or incorrect responses. Each response was followed by a $1000 \mathrm{~ms}$ intertrial interval (blank screen).
Following the completion of the memory experiment, participants were asked to return to their normal frame of mind and they were thoroughly debriefed concerning the purpose of research. Those participants who showed a continued response to the sad mood manipulation were cheered up by the experimenter to neutralise any increase in depression due to the sad mood induction procedure. Participants were encouraged to contact the experimenter in case they were experiencing any long-lasting sad mood. Each participant was contacted by phone after 1 week and 3 weeks. None of the participants reported any problem caused by our procedure.

\section{Data analysis}

A measure of the ability of participants to determine whether the probe replicated or not the head-pose orientation in the encoding display is provided by the sensitivity index $d^{\prime}$ used in signal detection theory. The statistic $d^{\prime}$ can be computed as $d^{\prime}=z$ (hit rate) $-z$ (false alarm rate). Equivalently, $d^{\prime}$ can be estimated by a probit regression (DeCarlo, 1998, 2010), ${ }^{3}$ with the advantage that, when the analysis is framed as a regression model, other predictor variables can be considered (Wright, Horry, \& Skagerberg, 2009). By following this second approach, we used a mixed-effect linear model with binomial error structure and a probit link function ${ }^{4}$ to analyse the participants' binary responses, with participants and items as crossed random effects, and Faceold (whether the probe was old or new) and Condition ("happy self-face", "happy nonself face", "sad self-face", "sad nonself face") as fixed effects. These analyses were performed using the lme4 package (Bates \& Sarkar, 2007) for the R statistical environment (version 2.15.1, R Core Team, 2012). We allowed the participants' accuracy to vary by adding to the random part of the

\footnotetext{
${ }^{3}$ Let $Y_{1}, \ldots, Y_{n}$ be the realisation of $n$ independent binary random variables, with probability of success $\operatorname{Pr}\left(Y_{i}=1\right)=\pi_{i}$. The $\pi_{i}$ are related to a set of $q$ predictors $x$. Let $x_{i j}$ denote the value of predictor $j(j=1, \ldots, q)$ for subject $i(i=1, \ldots, n)$. The probit regression model is $\pi_{i}=\Phi\left(\eta_{i}\right)$, where $\eta_{i}=\mathrm{S}_{j} \beta_{j} x$ ij called the linear predictor and $\Phi$ is the standard Gaussian cumulative distribution function.

${ }^{4}$ The link function $g()$ connects the expected value of the response variable, $\pi_{i}=E\left(Y_{i}\right), i=1, \ldots, n$, to the linear predictor: $g\left(\pi_{i}\right)=\eta_{i}$ and allows for a nonlinear relationship between $\pi_{i}$ and $\eta_{i}$. The probit link function transforms $\pi_{i}$ so that the regression curve for $\pi_{i}$ takes the form of the Gaussian cumulative distribution function.
} 
model both a term for the variance of accuracy and the covariance between accuracy and responding "old". An advantage of the mixedeffect analyses over the traditional approach (where an ANOVA is performed on the $d^{\prime}$ scores computed for each participant in each cell of the factorial design), is the possibility to specify crossed (or partially crossed) random effects for participants and items (see also Caudek, in press; Wright \& London, 2009). In this respect, mixed models can replace the by-subjects (F1) and byitems (F2) ANOVAs (Judd, Westfall, \& Kenny, 2012). Including additional random effects for items is especially important in the present case because a different set of pictures was used for each pair of participants.

We evaluated the effect of the variable Condition by computing the deviance statistics (minus 2 times the log-likelihood) of nested models; change in deviance is distributed as chi-square, with degrees of freedom equal to the number of parameters deleted from the model (e.g., Baayen, Davidson, \& Bates, 2008). In the presence of a statistically significant result, two planned comparisons ("self" vs. "nonself") were conducted: one for happy faces and one for sad faces. The per-comparison $\alpha$ was therefore set at .025 .

\section{RESULTS AND DISCUSSION}

\section{Participant profile}

Analysis of the participants' characteristics revealed that the four groups described in Table 1 did not differ significantly in terms of their age, $F(3,62)=0.31, p=.82$, or gender ratio, $\chi_{3}^{2}=4.69$, $p=.20$. Participants at "high cognitive risk" (hCRsR and hCRwR groups) scored significantly higher on the CSQ measure than did the participants at "low cognitive risk" (lCRsR and lCRwR groups), $M_{\text {high }}=4.18, M_{\text {low }}=3.14$, Welch $t(63.47)=9.88, \quad p=.001$. Participants with a strong response to sad MI (hCRsR and lCRsR groups) had higher VAS-dif scores than did participants with a weak response to sad MI (hCRwR and $1 \mathrm{CRwR}$ groups), $M_{\text {strong }}=6.91$, $M_{\text {weak }}=2.75$, Welch $t(61.44)=14.27, p=.001$.

Prior to testing Hypotheses 1 and 2, we verified that a negative mood had been induced in the same degree in the two groups classified as displaying a strong response to sad MI. The mean VAS-dif score did not significantly differ between the hCRsR group $(M=7.06, S E=0.27)$ and the lCRsR group $(M=6.78, S E=0.37)$, Welch $t(32.7)=0.72, p=.47$. Furthermore, the mean VAS-dif score did not significantly differ between the hCRwR group $(M=2.50, S E=0.26)$ and the lCRwR group $(M=3.05, S E=0.37)$, Welch $t(24.0)=1.22, p=.23$.

The four groups differed in terms of their average BDI-II, $F(3,62)=4.52, p=.006$, and RRS, $F(3,62)=5.31, p=.003$, scores. Post hoc comparisons using the Tukey HSD test indicated higher average scores on both variables in the hCRwR group (BDI: $M=13.88, S E=1.70$; RSS: $M=43.41, S E=2.94)$ relative to the $1 C R w R$ group (BDI: mean difference $=5.21, S E=1.16$; RSS: mean difference $=30.16, S E=1.42$ ). No other pairwise comparisons among the four groups were significant with respect to the mean BDI-II or RRS scores. The correlations among the BDI-II, CSQ, RRS, and VAS-dif scores are shown in Table 2.

\section{Effect of sad mood induction}

The mood induction produced significant changes in VAS self-rated sadness. Postinduction sadness was significantly higher than preinduction sadness: Mean change in mood was $49.6 \mathrm{~mm}$, $S D=24.0, t(65)=16.80, p=.001$.

Self-face advantage in head-pose recognition accuracy

When considering the full sample of 66 participants, a self-face processing advantage emerged for both happy faces (self-face: $d^{\prime}=2.01$, nonselfface: $\left.d^{\prime}=1.70, z=6.28, p=.001\right)$ and sad faces

TABLE 2

Correlation matrix among the BDI-II, CSQ, RRS, and VAS-dif scores

\begin{tabular}{lcccc}
\hline & BDI-II & $C S Q$ & $R R S$ & VAS-dif \\
\hline BDI-II & 1.0 & & & \\
CSQ & $.37(.01)$ & 1.0 & & \\
RRS & $.82(.001)$ & $.35(.02)$ & 1.0 & \\
VAS-dif & $-.23(.13)$ & $-.08(.52)$ & $-.35(.01)$ & 1.0 \\
\hline
\end{tabular}

BDI-II is Beck Depression Inventory Second Edition. CSQ is Cognitive Style Questionnaire. RRS is Ruminative Response Scale. VAS-dif is the difference between the postinduction and the preinduction mood checks measured with the Visual Analogue Scale. The values shown in the parentheses are the probability values adjusted for multiple tests. 
(self-face: $d^{\prime}=1.93$, nonself face: $d^{\prime}=1.62, z=$ $6.61, p=.001)^{5}$ - see Figure 2. This result is consistent with previous findings (Keenan, Wheeler, Gallup, \& Pascual-Leone, 2000; Keyes \& Brady, 2010).

The novel result of the present study is that the self-face advantage is modulated by (1) facial affect, (2) negative cognitive style, and (3) response to sad mood induction. Figure 3 shows recognition performance for head-pose orientation as a function of condition ("happy self-face", "happy nonself face", "sad self-face", "sad nonself face") in the four groups of participants. Although overall average accuracy did not vary across groups $\left(d^{\prime}{ }_{\mathrm{hCRsR}}=1.81, \quad d^{\prime}{ }_{\mathrm{hCRwR}}=1.89\right.$, $\left.d^{\prime}{ }_{1 \mathrm{CRsR}}=1.83, d^{\prime}{ }_{\mathrm{lCRwR}}=1.82\right), \chi_{3}^{2}=0.04, p=.99,{ }^{6}$ there was a statistically significant Condition $\times$ Group interaction, $\chi_{9}^{2}=31.80, p=.001$. Hence, the effects of condition on recognition accuracy were examined separately in each group.

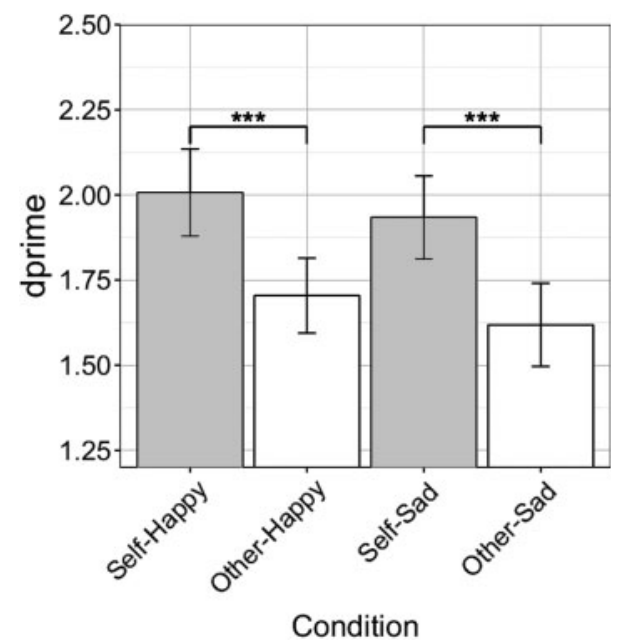

Figure 2. Sensitivity for head-pose (orientation) recognition as a function of Condition (self happy, other happy, self sad, other sad) in the whole sample of 66 participants. Error bars indicate \pm 1 standard error. $* p<.05, * * p<.01, * * * p<.001$.

\footnotetext{
${ }^{5}$ When computing $d^{\prime}$ separately for each participant and each condition with traditional Signal Detection Theory methods, we obtained the following mean sensitivity values in the full sample of 66 participants: $d_{\text {self happy }}^{\prime}=1.99, d_{\text {nonself }}^{\prime}$ happy $=1.69, d_{\text {self sad }}^{\prime}=1.92, d_{\text {nonself sad }}^{\prime}=1.58$. These values are almost identical to those found with a multilevel model: $d_{\text {self }}^{\prime}$ happy $=2.01, d_{\text {nonself happy }}^{\prime}=1.70, d_{\text {self sad }}^{\prime}=1.93, d_{\text {nonself sad }}^{\prime}=$ 1.62 (for a discussion, see Wright \& London, 2009). Importantly, the addition of a random intercept for items significantly improves model fit, $\chi_{1}^{2}=11.35, p=.001$.

${ }^{6}$ Analysis of response bias did not yield significant group differences in terms of criterion $C, F(3,62)=0.31, p=.82$, or false alarm rates, $F(3,62)=0.282, p=.84$.
}

Participants at high cognitive risk with a strong response to sad MI. Memory for head-pose orientation was modulated by condition, $\chi_{6}^{2}=40.37, p=.001$. Participants were more accurate responding to the self-face than to the nonself face for sad facial expressions, $\Delta d^{\prime}=$ $0.52, z=5.59, p=.001$. No difference in recognition accuracy between self and nonself faces was found for happy facial expressions, $\Delta d^{\prime}=0.16, z=$ $1.74, p=.082$.

Participants at high cognitive risk with a weak response to sad MI. Memory for head-pose orientation was modulated by condition, $\chi_{6}^{2}=17.34, p=.001$. Participants were more accurate responding to the self-face than to the nonself face for happy facial expressions, $\Delta d^{\prime}=$ $0.25, z=2.65, p=.001$. No difference in recognition accuracy between self and nonself faces was found for sad facial expressions, $\Delta d^{\prime}=0.10, z=$ $1.11, p=.268$.

Participants at low cognitive risk with a strong response to sad MI. Memory for head-pose orientation was modulated by condition, $\chi_{6}^{2}=71.25, p=.001$. A self-face advantage was found for happy expressions, $\Delta d^{\prime}=0.66, z=6.87$, $p=.001$, and for sad expressions, $\Delta d^{\prime}=0.37, z=$ $4.01, p=.001$. However, the self-face advantage was stronger for happy expressions than for sad expressions, $\chi_{1}^{2}=4.31, p=.038$.

Participants at low cognitive risk with a weak response to sad MI. Memory for head-pose orientation was not modulated by condition, $\chi_{6}^{2}=8.06, p=.234$.

A separate analysis indicated that the size of the self-face advantage (i.e., the $d$ ' difference in head-pose orientation recognition between the self-face and a stranger's face) varied across the four groups described above, $\chi_{3}^{2}=16.58, p=.001$. For the group represented in Figure $3 \mathrm{c}$, the selfface advantage was significantly larger than for the groups represented in Figures $3 \mathrm{a}, \Delta d^{\prime}=0.19$, $z=2.06, p=.040,3 \mathrm{~b}, \Delta d^{\prime}=0.33, z=3.47, p=.001$, and $3 \mathrm{~d}, \Delta d^{\prime}=0.38, z=3.87, p=.001$.

\section{Self-face advantage in the reaction times}

Previous research has shown that observers respond faster to their own face than to unfamiliar faces (Keenan et al., 2000; Tong \& Nakayama, 1999). We replicated this finding: On average, response latencies to the self-face were $35 \mathrm{~ms}$ 


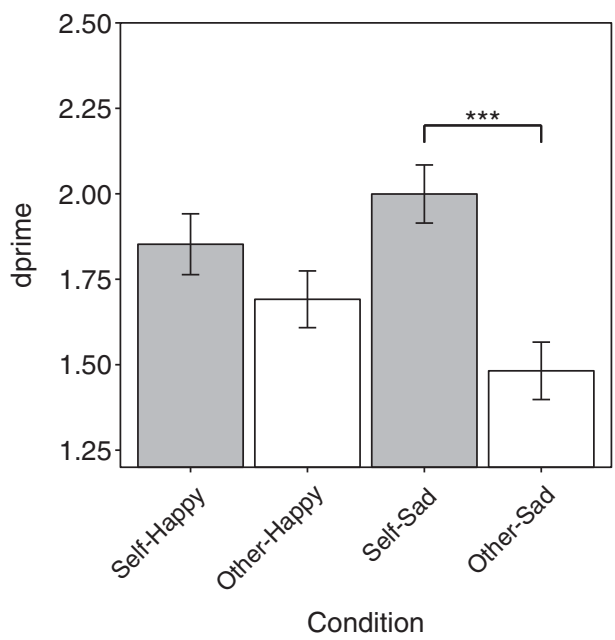

(a) Group: hCRsR

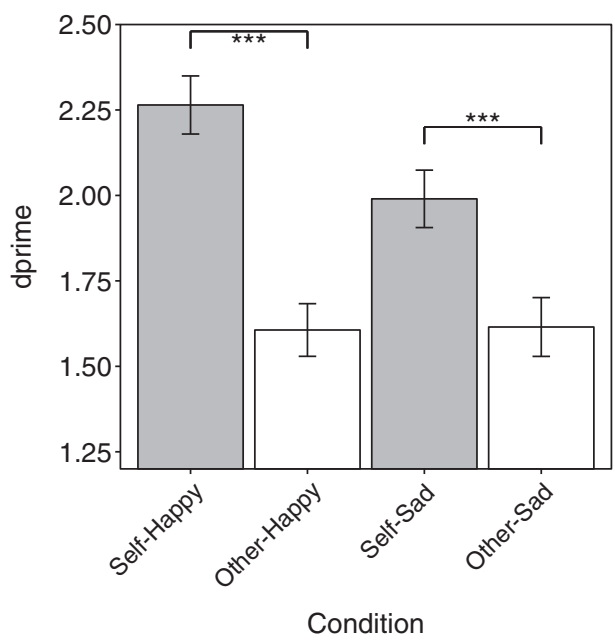

(c) Group: ICRsR

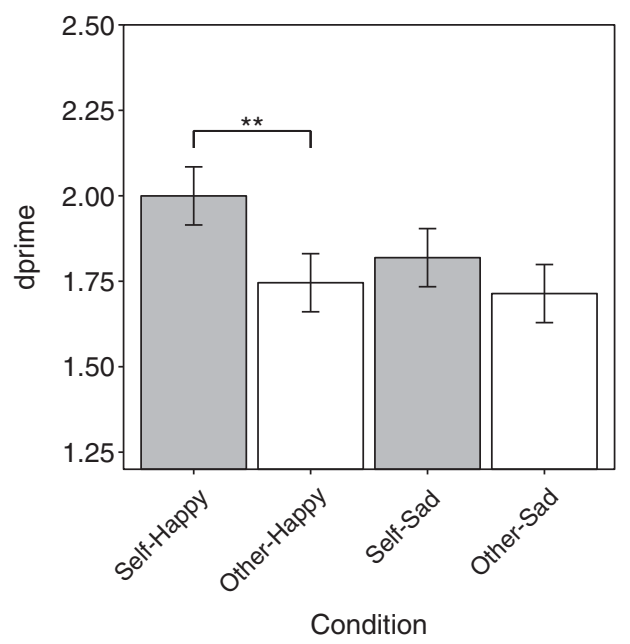

(b) Group: hCRwR

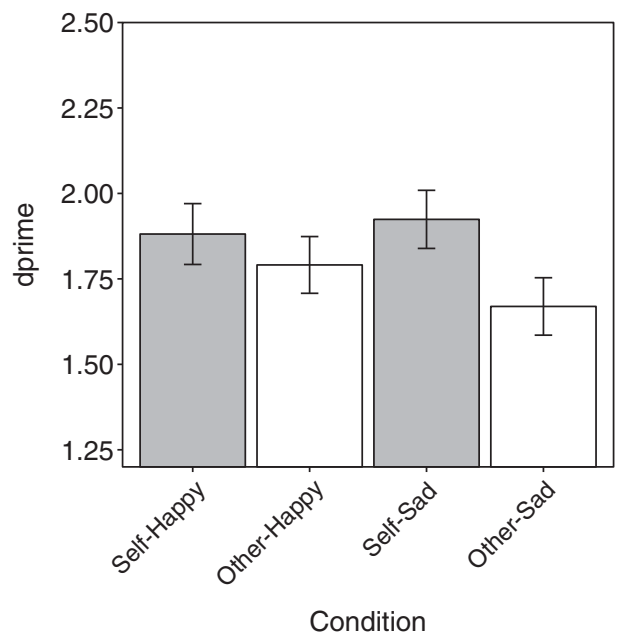

(d) Group: ICRwR

Figure 3. $d^{\prime}$ sensitivity for head-pose (orientation) recognition as a function of Condition (self happy, other happy, self sad, other sad) in each group. $h C R s R$ : participants at high cognitive risk with a strong response to sad mood induction; $h C R w R$ : participants at high cognitive risk with a weak response to sad mood induction; $l C R s R$ : participants at low cognitive risk with a strong response to sad mood induction; $l C R w R$ : participants at low cognitive risk with a weak response to sad mood induction. Error bars indicate \pm 1 standard error. $* p<.05, * * p<.01, * * * p<.001$.

faster than response latencies to unfamiliar faces, $\chi_{1}^{2}=11.40, p=.002$. Response times were not affected by group (hCRsR, hCRwR, lCRsR, lCRwR), by valence (happy, sad), or by their interaction, $\chi_{14}^{2}=10.88, p=.69$.

\section{Self-face advantage and alternative} classifications of participants

To further evaluate the hypotheses described earlier, we created for comparison three alternative classifications of participants. In a first classification, two extreme groups were created by dividing participants into quartiles based on their
CSQ scores, regardless of their response to the sad MI. In a second classification, two extreme groups were created by dividing participants into quartiles based on their response to the sad MI, regardless of their CSQ scores. A further classification of participants was based on their BDI-II scores. In all cases, only participants in the highest and lowest quartiles were retained for further analysis. The mean scores for the high and low quartile groups were $4.47(S E=0.10, n=16)$ and $2.80(S E=0.09$, $n=16)$ for the CSQ split, $7.95(S E=0.19, n=16)$ and $1.74(S E=0.09, n=16)$ for the VAS-dif split, and $20.25(S E=1.48, n=16)$ and $1.78(S E=0.31$, $n=18$ ) for the BDI-II split, respectively. 
Statistical analyses were performed as described previously, and significant differences for self versus nonself face comparisons are reported in Table $3(\alpha=.025)$. The main result of these analyses is that none of these alternative classification criteria was able to discriminate between participants who displayed a maladaptive WM bias for the self-face from those who did not.

\section{Sad benefit in head-pose recognition accuracy}

Earlier, we compared the recognition accuracy between the self and nonself face, for different expressions. In a further set of analyses, we compared the recognition accuracy between the happy and sad expressions. With these analyses, we found no evidence of a sad benefit in any group of participants. On the contrary, we found an advantage for happy faces over sad faces when considering the nonself face pictures in the group of participants at high cognitive risk with a strong response to sad MI, $\Delta d^{\prime}=0.21, z=2.31, p=.021$, and when considering the self-face pictures in the group of participants at low cognitive risk with a strong response to sad MI, $\Delta d^{\prime}=0.27, z=2.80$, $p=.005$.

TABLE 3

Recognition sensitivity $\left(d^{\prime}\right)$ for head-pose (orientation) as a function of condition (happy self-face, happy nonself face, sad self-face, sad nonself face)

\begin{tabular}{lccc}
\hline & CSQ & VAS-dif & BDI-II \\
\hline High quartile group & & & \\
Happy self-face & $\mathbf{1 . 9 6}(0.06)$ & $\mathbf{2 . 1 6}(0.10)$ & $\mathbf{1 . 5 6}(0.08)$ \\
Happy nonself-face & $\mathbf{1 . 7 2}(0.06)$ & $\mathbf{1 . 7 6}(0.08)$ & $\mathbf{1 . 3 2}(0.07)$ \\
Sad self-face & $\mathbf{1 . 8 8}(0.06)$ & $\mathbf{2 . 1 6}(0.09)$ & $\mathbf{1 . 4 5}(0.08)$ \\
Sad nonself-face & $\mathbf{1 . 6 6}(0.06)$ & $\mathbf{1 . 6 3}(0.08)$ & $\mathbf{1 . 2 2}(0.08)$ \\
Low quartile group & & & \\
Happy self-face & $\mathbf{2 . 0 3}(0.10)$ & $\mathbf{2 . 0 5}(0.09)$ & $\mathbf{2 . 2 0}(0.09)$ \\
Happy nonself-face & $\mathbf{1 . 6 3}(0.09)$ & $\mathbf{1 . 7 3}(0.08)$ & $\mathbf{1 . 7 5}(0.07)$ \\
Sad self-face & $1.89(0.09)$ & $1.85(0.08)$ & $\mathbf{2 . 0 3}(0.08)$ \\
Sad nonself-face & $1.72(0.09)$ & $1.70(0.10)$ & $\mathbf{1 . 6 3}(0.09)$ \\
\hline
\end{tabular}

Classifications of participants are based on upper and lower quartile splits on CSQ, VAS-dif, or BDI-II scores. BDIII is Beck Depression Inventory Second Edition. CSQ is Cognitive Style Questionnaire. VAS-dif is the difference between the postinduction and the preinduction mood checks measured with the Visual Analogue Scale. Statistically significant differences between the self and the nonself conditions are highlighted in bold. When the self-face effect was statistically significant for both happy and sad faces, we found no statistical evidence that its size differed across happy and sad faces within any group defined by these alternative classifications. Standard errors are reported in parentheses.

\section{GENERAL DISCUSSION}

In our study, healthy volunteers performed a head-pose (orientation) recognition task. The stimuli depicted either the participant's own face or unknown faces, with happy or sad expressions (Figure 1). We found a strong self-reference effect (i.e., better head-pose recognition for the self-face than for stranger's faces), but also marked individual differences. Some participants displayed a self-face advantage for sad expressions, whereas others displayed a stronger self-face advantage for happy expressions. Consistent with diathesisstress theories, these individual differences were associated with trait-like factors (negative cognitive style) and affective states (response to sad mood induction).

- Participants who scored above the sample median on both CSQ and VAS-dif displayed a self-face advantage for sad but not happy faces (Figure 3a). This result indicates that, when experiencing an affective challenge (strong sad mood induction), participants high in negative cognitive style display a negative self-referential memory bias (Hypothesis 1).

- Participants who scored above the sample median on CSQ but below the median on VAS-dif displayed a self-face advantage for happy but not sad faces (Figure 3b). When not distressed, participants high in negative cognitive style do not exhibit a negative selfreferential bias (Hypothesis 2). This result is in line with schema models, which postulate that latent negative schemas becomes activated only in distressing situations (Clark, Beck, \& Alford, 1999; Ingram, 1984), but not with impaired cognitive control accounts, which postulate that cognitive biases operate as long as the cognitive control impairments persist, regardless of a negative mood state (see Joormann, Talbot, \& Gotlib, 2007).

- Participants who scored below the sample median on CSQ and above the median on VAS-dif displayed a stronger self-face advantage for happy faces than for sad faces (Hypothesis 3) - see Figure 3c. This result is consistent with an asymmetric recall favouring positive information among psychologically resilient individuals (Matt et al., 1992). Note, moreover, that the self-face advantage was stronger for the group of participants 
represented in Figure $3 \mathrm{c}$ than for the other three groups (Figures 3a, 3b, and 3d). This result is in agreement with previous studies showing that sad mood, alone and/or in combination with arousal, increases selffocused attention in healthy individuals (e.g., Panayiotou, Brown, \& Vrana, 2007). ${ }^{7}$

- Finally, participants who scored below the sample median on both CSQ and VASdif displayed no self-face advantage at all (Figure 3d). Previous research has indicated effects of state affect on information processing style. ${ }^{8}$ The results reported in Figures $3 \mathrm{c}$ and $3 \mathrm{~d}$ indicate that mood level can modulate the self-face effect, also when latent negative schemas are absent.

Emotional faces have been used to study the mood-congruent memory bias in patients with depression and in individuals with sub-clinical dysphoria or induced sad mood. A long-term memory bias for sad faces has been found in patients with depression (Gilboa-Schechtman, Erhard-Weiss, \& Jeczemien, 2002; Ridout, Astell, Reid, Glen, \& O'Carroll, 2003), in individuals experiencing dysphoria (Jermann et al., 2008; Ridout, Noreen, \& Johal, 2009), and in individuals in an induced sad mood (Ridout, Dritschel, et al., 2009). A short-term memory bias for sad faces has been found in patients with melancholic depression (Linden, Jackson, Subramanian, Healy, \& Linden, 2011), but not in individuals with subclinical dysphoria (Noreen \& Ridout, 2010). The results of these studies show abnormal recognition memory for faces displaying sadness in depressed and depression-susceptible groups, or after an experimentally induced sad mood. The present study adds to previous findings by considering not only the facial affect, but also the distinction between the self-face and the nonself face. We showed that individuals high in negative

\footnotetext{
${ }^{7}$ For example, Sedikides (1992) found that participants in whom a sad mood had been induced produced a larger number of references to the self when asked to tell a story than those in whom a happy or neutral mood had been induced. Wood, Saltzberg, and Goldsamt (1990) found that, in a sentence-completion task, participants induced to feel sad produced a higher number of first-person singular pronouns than those induced to feel happy or neutral.

${ }^{8}$ Positive mood is associated with using stereotypes and global processing, whereas negative mood is associated with greater accuracy, lower false positive recognition, resistance to heuristic error, and local processing (e.g., Gasper \& Clore, 2001).
}

cognitive style, when distressed, show a bias towards the negative self-face (with respect to the negative nonself face), even within brief short-term memory episodes. Conversely, we found no evidence of a "sad benefit" during WM processing (see also Noreen \& Ridout, 2010).

One methodological novelty of the present study is the use of pictures of the participant's own face for the study of emotional face processing in WM. A processing advantage for the selfface has been found in many behavioural tasks. Participants respond faster to their own faces than to faces of unfamiliar or familiar others in visual search (Tong \& Nakayama, 1999). For the selfface, performance is better in face identification tasks (Keenan et al., 1999) and in head orientation identification tasks (Ma \& Han, 2010; Sui \& Han, 2007). Moreover, self-referential encoding strategies enhance memory to a greater degree than other encoding strategies (Grilli \& Glisky, 2010; Kesebir \& Oishi, 2010). Turk, Cunningham, and Macrae (2008) showed that the self-reference effect emerges also in the case of an incidental pairings between the photographic image of the participant's own face and the target words, without any direct evaluative processing of the face images. By using a head-pose recognition task, we extended these findings by showing that the self-reference effect emerges also under conditions of incidental association between headpose orientation and face identity. Moreover, we showed that the incidental associations between self and target information can impact on memory performance in opposite ways, depending on facial affect and cognitive style.

The biases illustrated in Figure 3 cannot be accounted for by differences in cognitive impairment, because the four groups did not differ in overall memory performance or in the average latency of the participants' responses. Moreover, the results of Figure 3 cannot be attributed to differences in rumination (Raes, Hermans, \& Williams, 2006) or dysphoric affect. We found higher RRS and BDI-II scores in the hCRwR group than in the lCRsR group, but this does not explain why a maladaptive WM bias was only found in the hCRsR group.

A few limitations of this study along with questions for future research should be noted. (1) From the present data we cannot determine whether the WM biases for emotional faces illustrated in Figure 3 depend on selective attention at encoding and/or on biased retrieval 
processes. (2) Although we have operationalised the negative cognitive style with the CSQ scores, it would be interesting to complement the present results with other measures such as the Dysfunctional Attitudes Scale (DAS; Weissman \& Beck, 1978). (3) Participants selection could be improved so as to create more extreme groups. (4) The analyses described earlier could benefit by use of the Center for Epidemiologic Studies Depression Scale (CES-D) rather than the BDIII scale. (5) Our task involved a decision concerning the head-pose orientation of the probe face, whereas the emotional valence and the identity of the faces were irrelevant to the task. The incidental encoding of facial affect had the purpose of activating unintentionally automatic and schema-driven responses, independent of deliberative, explicit processes (e.g., Haeffel et al., 2007). Future research could establish how the results vary if participants are asked to explicitly process the emotional valence or the identity of the faces during the encoding phase. ${ }^{9}$ (6) Future research could also establish how the results vary if participants are asked to perform a long-term memory task. (7) The four groups of participants likely differed also on other characteristics. Thus, more research is needed to establish whether other variables (e.g., personality or temperament) may have contributed to the current results. (8) Gotlib and Joormann (2010) remarked that only few investigations have tested the diathesis-stress model by assessing biased processing prior to the first onset of depression. This is exactly what we have done here. However, they also pointed out that this assessment represents only a first step to establish whether biased processing can predict depression following the experience of a negative life event. This question needs to be addressed in future research, together with the study of negative cognitions about the self face in clinically depressed individuals.

In conclusion, our study indicates that negative mood, when experienced by individuals high in negative cognitive style, can activate a maladaptive WM bias in which the self-face is remembered better than a stranger's face, but only for sad expressions. When tested under conditions that do not provoke their vulnerability, individuals high in negative cognitive style do not

\footnotetext{
${ }^{9}$ For a discussion of the effects of an explicit versus implicit encoding of the emotional content of the to-be-remembered stimuli on the mood-congruent memory bias, see Ridout, Dritschel, et al. (2009) and Wisco (2009).
}

display this WM bias, and neither do individuals low in negative cognitive style.

Manuscript received 21 February 2012
Manuscript accepted 7 January 2013
First published online 5 February 2013

\section{REFERENCES}

Abramson, L. Y., Alloy, L. B., Hankin, B. L., Haeffel, G. J., MacCoon, D. G., \& Gibb, B. E. (2002). Cognitive vulnerability-stress models of depression in a self-regulatory and psychobiological context. In I. H. Gotlib \& C. L. Hammen (Eds.), Handbook of depression (1st ed., pp. 268-294). New York, NY: Guilford Press.

Abramson, L. Y., Metalsky, G. I., \& Alloy, L. B. (1989). Hopelessness depression: A theory-based subtype of depression. Psychological Review, 96, 358-372.

Alloy, L. B., Abramson, L. Y., Hogan, M. E., Whitehouse, W. G., Rose, D. T., Robinson, M. S., ... Lapkin, J. B. (2000). The Temple-Wisconsin Cognitive Vulnerability to Depression Project: Lifetime history of Axis I psychopathology in individuals at high and low cognitive vulnerability to depression. Journal of Abnormal Psychology, 109, 403-418.

Alloy, L. B., Abramson, L. Y., Keyser, J., Gerstein, R. K., \& Sylvia, L. G. (2008). Negative cognitive style. In K. S. Dobson \& D. J. A. Dozois (Eds.), Risk factors in depression (pp. 237-262). San Diego, CA: Elsevier Academic Press.

Baayen, R. H., Davidson, D. J., \& Bates, D. M. (2008). Mixed-effects modeling with crossed random effects for subjects and items. Journal of Memory and Language, 59, 390-412.

Bates, D., \& Sarkar, D. (2007). Ime4. Linear mixedeffects models using $S 4$ classes (R package Version 0.9975) [Computer software]. Available from http:// cran.r-project.org/web/pakages/lme4/index.html

Beck, A. T., Steer, R. A., \& Brown, G. K. (1996). Beck Depression Inventory (2nd ed.). San Antonio, TX: Psychological Corporation.

Bistricky, S. L., Ingram, R. E., \& Atchley, R. A. (2011). Facial affect processing and depression susceptibility: Cognitive biases and cognitive neuroscience. Psychological Bulletin, 137, 998-1028.

Caudek, C. (in press). The fidelity of visual memory for faces and non-face objects. Acta Psychologica. doi:10.1016/j.actpsy.2012.12.002

Clark, D. A., Beck, A. T., \& Alford, B. A. (1999). Scientific foundations of cognitive theory and therapy of depression. New York, NY: Wiley.

DeCarlo, L. T. (1998). Signal detection theory and generalized linear models. Psychological Methods, 3 , 186-205.

DeCarlo, L. T. (2010). On the statistical and theoretical basis of signal detection theory and extensions: Unequal variance, random coefficient and mixture models. Journal of Mathematical Psychology, 54, 304-313.

Disner, S. G., Beevers, C. G., Haigh, E. A. P., \& Beck, A. T. (2011). Neural mechanisms of the cognitive 
model of depression. Nature Reviews Neuroscience, 12, 467-477.

Forgas, J. P., \& East, R. (2008). How real is that smile? Mood effects on accepting or rejecting the veracity of emotional facial expressions. Journal of Nonverbal Behavior, 32, 157-170.

Gasper, K., \& Clore, G. L. (2002). Attending to the big picture: Mood and global versus local processing of visual information. Psychological Science, 13, 34-40.

Ghisi, M., Flebus, G. B., Montano, A., Sanavio, E., \& Sica, C. (2006). Beck Depression Inventory-II: Manuale. Firenze, Italy: Organizzazioni Speciali.

Gilboa-Schechtman, E., Erhard-Weiss, D., \& Jeczemien, P. (2002). Interpersonal deficits meet cognitive biases: Memory for facial expressions in depressed and anxious men and women. Psychiatry Research, 113, 279-293.

Gotlib, I. H., \& Joormann, J. (2010). Cognition and depression: Current status and future direction. Annual Review of Clinical Psychology, 27, 285-312.

Grilli, M. D., \& Glisky, E. L. (2010). Self-imagining enhances recognition memory in memory-impaired individuals with neurological damage. Neuropsychology, 24, 698-710.

Haeffel, G. J., Abramson, L. Y., Brazy, P. C., Shah, J. Y., Teachman, B. A., \& Nosek, B. A. (2007). Explicit and implicit cognition: A preliminary test of a dual process theory of cognitive vulnerability to depression. Behaviour Research and Therapy, 45, 1155-1167.

Haeffel, G. J., Gibb, B. E., Metalsky, G. I., Alloy, L. B., Abramson, L. Y., Hankin, B. L., \& ... Swendsen, J. D. (2008). Measuring cognitive vulnerability to depression: Development and validation of the Cognitive Style Questionnaire. Clinical Psychology Review, 28, 824-836.

Hedlund, S., \& Rude, S. S. (1995). Evidence of latent depressive schemas in formerly depressed individuals. Journal of Abnormal Psychology, 104, 517-525.

Hertel, P. T. (2004). Memory for emotional and nonemotional events in depression: A question of habit? In D. Reisberg \& P. Hertel (Eds.), Memory and emotion (pp. 186-216). New York, NY: Oxford University Press.

Hewig, J., Hagemann, D., Seifert, J., Gollwitzer, M., Naumann, E., \& Bartussek, D. (2005). Brief report. Cognition and Emotion, 17, 1095-1109.

Ingram, R. E. (1984). Toward an information-processing analysis of depression. Cognitive Therapy and Research, 8, 443-477.

Ingram, R. E., Atchley, R. A., \& Segal, Z. V. (2011). Vulnerability to depression: From cognitive neuroscience to prevention and treatment. New York, NY: Guilford Press.

Jallais, C., \& Gilet, A. L. (2010). Inducing changes in arousal and valence: Comparison of two mood induction procedures. Behaviour Research and Methods, 42, 318-325.

Jermann, F., Van der Linden, M., \& D'Argembeau, A. (2008). Identity recognition and happy and sad facial expression recall: Influence of depressive symptoms. Memory, 16, 364-373.

Joormann, J., Talbot, L., \& Gotlib, I. H. (2007). Biased processing of emotional information in girls at risk for depression. Journal of Abnormal Psychology, 116, 135-143.

Judd, C. M., Westfall, J., \& Kenny, D. A. (2012). Treating stimuli as a random factor in social psychology: A new and comprehensive solution to a pervasive but largely ignored problem. Journal of Personality and Social Psychology, 103, 54-69.

Keenan, J. P., McCutcheon, B., Freund, S., Gallup, G. G. Jr., Sanders, G., \& Pascual-Leone, A. (1999). Left hand advantage in a self-face recognition task. Neuropsychologia, 37, 1421-1425.

Keenan, J. P., Wheeler, M. A., Gallup, G. G. Jr., \& Pascual-Leone, A. (2000). Self-recognition and the right prefrontal cortex. Trends in Cognitive Science, 4, 338-344.

Kesebir, S., \& Oishi, S. (2010). A spontaneous selfreference effect in memory: Why some birthdays are harder to remember than others. Psychological Science, 21, 1525-1531.

Keyes, H., \& Brady, N. (2010). Self-face recognition is characterized by "bilateral gain" and by faster, more accurate performance which persists when faces are inverted. Quarterly Journal of Experimental Psychology, 63, 840-847.

Linden, S. C., Jackson, M. C., Subramanian, L., Healy, D., \& Linden, D. E. J. (2011). Sad benefit in face working memory: An emotional bias of melancholic depression. Journal of Affective Disorders, 135, 251-257.

Lovell, D. (Prod.), \& Zeffirelli, F. (Dir.). (1979). The Champ [Motion picture]. USA: MGM/Pathe Home Video.

Ma, Y., \& Han, S. (2009). Self-face advantage is modulated by social threat-Boss effect on self-face recognition. Journal of Experimental Social Psychology, 45, 1048-1051.

Ma, Y., \& Han, S. (2010). Why we respond faster to the self than to others? An implicit positive association theory of self-advantage during implicit face recognition. Journal of Experimental Psychology: Human Perception and Performance, 36, 619-633.

Matt, G. E., Vazquez, C., \& Campbell, W. K. (1992). Mood-congruent recall of affectively toned stimuli: A meta-analytic review. Clinical Psychology Review, 12, 227-255.

McCormack, H., Horne, D., \& Sheather, S. (1988). Clinical applications of visual analogue scales: A critical review. Psychological Medicine, 18, 1007-1019.

Nolen-Hoeksema, S., \& Morrow, J. (1993). Effects of rumination and distraction on naturally occurring depressed mood. Cognition and Emotion, 7, 561-570.

Noreen, S., \& Ridout, N. (2010). Short-term memory for emotional faces in dysphoria. Memory, 18, 486-497.

Palmieri, R., Gasparre, A., \& Lanciano, T. (2007). Una misura disposizionale della Ruminazione depressiva: La RRS di Nolen-Hoeksema e Morrow. Psychofenia, 10, 15-33.

Panayiotou, G., Brown, R., \& Vrana, S. R. (2007). Emotional dimensions as determinants of selffocused attention. Cognition and Emotion, 21, 982-998.

Raes, F., Hermans, D., \& Williams, J. M. (2006). Negative bias in the perception of others' facial emotional expressions in major depression: The role 
of depressive rumination. Journal of Nervous and Mental Disease, 194, 796-799.

R Core Team. (2012). R: A language and environment for statistical computing. R Foundation for Statistical Computing, Vienna, Austria. ISBN 3-900051-07-0. Available from http://www.R-project.org/

Ramel, W., Goldin, P. R., Eyler, L. T., Brown, G. G., Gotlib, I. H., \& McQuaid, J. R. (2007). Amygdala reactivity and mood-congruent memory in individuals at risk for depressive relapse. Biological Psychiatry, 61, 231-239.

Ridout, N., Astell, A. J., Reid, I. C., Glen, T., \& O'Carroll, R. E. (2003). Memory bias for emotional facial expressions in major depression. Cognition and Emotion, 17, 101-122.

Ridout, N., Dritschel, B., Matthews, K., McVicar, M., Reid, I. C., \& O'Carroll, R. E. (2009). Memory for emotional faces in major depression following judgement of physical facial characteristics at encoding. Cognition and Emotion, 23, 739-752.

Ridout, N., Noreen, A., \& Johal, J. (2009). Memory for emotional faces in naturally occurring dysphoria and induced sadness. Behaviour Research and Therapy, 47, 851-860.

Romens, S. E., MacCoon, D. G., Abramson, L. Y., \& Pollak, S. D. (2011). Cognitive style moderates attention to attribution-relevant stimuli. Cognitive Therapy and Research, 35, 134-141.

Rowe, G., Hirsh, J. B., \& Anderson, A. K. (2007). Positive affect increases the breadth of attentional selection. Proceedings of the National Academy of Sciences, 104, 383-388.

Scher, C. D., Ingram, R. E., \& Segal, Z. V. (2005). Cognitive reactivity and vulnerability: Empirical evaluation of construct activation and cognitive diathesis in unipolar depression. Clinical Psychology Review, 25, 487-510.

Scherrer, M. C., \& Dobson, K. S. (2009). Predicting responsiveness to a depressive mood induction procedure. Journal of Clinical Psychology, 65, 20-35.

Sedikides, C. (1992). Mood as a determinant of attentional focus. Cognition and Emotion, 6, 129-148.

Sica, C., Caudek, C., Chiri, L. R., Ghisi, M., \& Marchetti, I. (2012). Not just right experiences" predict obsessive-compulsive symptoms in non-clinical Italian individuals: A one-year longitudinal study. Journal of Obsessive-Compulsive and Related Disorders, 1, 159-167.

Sica, C., \& Ghisi, M. (2007). The Italian versions of the Beck anxiety inventory and the Beck Depression
Inventory-II: Psychometric properties and discriminant power. In M. A. Lange (Ed.), Leading-edge psychological tests and testing (pp. 27-50). New York, NY: Nova.

Singer, A. R., \& Dobson, K. S. (2007). An experimental investigation of the cognitive vulnerability to depression. Behaviour Research and Therapy, 45, 563-575.

Sui, J., \& Han, S. (2007). Self-construal priming modulates neural substrates of self-awareness. Psychological Science, 18, 861-866.

Tong, F., \& Nakayama, K. (1999). Robust representations for faces: Evidence from visual search. Journal of Experimental Psychology: Human Perception and Performance, 25, 1016-1035.

Turk, D. J., Cunningham, S., \& Macrae, N. (2008). Selfmemory biases in explicit and incidental encoding of trait adjectives. Consciousness and Cognition, 17, 1040-1045.

Watkins, E., Teasdale, J. D., \& Williams, R. M. (2003). Contextual questions prevent mood primes from maintaining experimentally induced dysphoria. Cognition and Emotion, 17, 455-475.

Watson, L. A., Dritschel, B., Obonsawin, M. C., \& Jentzsch, I. (2007). Seeing yourself in a positive light: Brain correlates of the self-positivity bias. Brain Research, 1152, 106-110.

Weissman, A. N., \& Beck, A. T. (1978). Development and validation of the Dysfunctional Attitude Scale. Paper presented at the annual meeting of the Association for the Advancement of Behavior Therapy, Chicago, IL.

Werner-Seidler, A., \& Moulds, M. L. (2012). Mood repair and processing mode in depression. Emotion, $12,470-478$.

Wisco, B. E. (2009). Depressive cognition: Self-reference and depth of processing. Clinical Psychology Review, 29, 382-392.

Wood, J. V., Saltzberg, J. A., \& Goldsamt, L. A. (1990). Does affect induce self-focused attention? Journal of Personality and Social Psychology, 58, 899-908.

Wright, D. B., Horry, R., \& Skagerberg, E.M. (2009). Functions for traditional and multilevel approaches to signal detection theory. Behaviour Research Methods, 41, 257-267.

Wright, D. B., \& London, K. (2009). Multilevel modelling: Beyond the basic applications. British Journal of Mathematical and Statistical Psychology, 62, 439-456. 
Copyright of Memory is the property of Psychology Press (UK) and its content may not be copied or emailed to multiple sites or posted to a listserv without the copyright holder's express written permission. However, users may print, download, or email articles for individual use. 